\title{
Analysis and Design of High Performance Ring Voltage Controlled Oscillator
}

\author{
Ashraf Mohamed Ali Hassen \\ Department of Electronics and Communications \\ Modern Academy in Maadi
}

\begin{abstract}
The voltage controlled oscillator (VCO) may be considered one of the most important building blocks in modern communication applications such as microprocessor clock generation, wired and wireless communications, system synchronization, and frequency synthesis. The search in the field of design for high performance VCOs has been increasingly more important and becomes an active research area. In the past decade, The Researches on VCOs have been based on the areas of higher frequency, lower phase noise, low power, low operating voltage, and increased tuning range. However many of these objectives can be only achieved at the expense of some other objectives. This thesis analyzes the design of high performance ring VCOs. The beginning of the thesis is reviewing the basic ring VCOs. The different designs are also introduced. Finally, the circuit techniques used in a proposed VCO based on the new technique is designed and simulated in $65 \mathrm{~nm}$ CMOS SOI process. The results of proposed device are compared and confirmed the usability of the new ring VCO cell topology. Finally, a conclusion of the proposed design of high performance ring VCOs is explained.
\end{abstract}

\section{General Terms}

Voltage Controlled oscillator - Clock Generation - Wireless Communications.

\section{Keywords}

Phase noise, power, operating voltage, tuning range

\section{INTRODUCTION}

A VCO is the most important basic building blocks in analog and digital circuits [1]. There are various implementations of VCOs. One of them is a ring oscillator based on VCO, which is commonly used in the clock generation subsystem. The main reason of using ring oscillator in different fields of communications system is a direct consequence of its easy integration. Due to their integrated nature, ring oscillators have become one of the most important building block in many digital and communication systems. The most applications of voltage controlled oscillators are recovery circuits for serial data communications [1,2] disk-drive read channels [3] on-chip clock distribution [4], and integrated frequency synthesizers $[5,6]$. There are many tradeoffs in the design of a ring oscillator in terms of speed, power, area, and application domain [7]. The problem of designing a ring oscillator is the focus of interest in this paper. In the communication system, the voltage controlled oscillator is the important component, especially in the PLL circuit, clock recovery circuit and frequency integrated circuit, so it is the top priorities [8].

Over the last several decades, the rapid growth of commercial communication applications has led to a commensurate demand for better and cheaper devices. While Moore's law has continued its course mostly undisturbed for close to a half century, digital circuits have experienced continuing improvements due to a doubling of available transistors every two years $[9,10]$. Such rapid technology developments revolutionized digital electronics, there by fueling one of the fastest growing markets ever observed.

Analog circuits have also controlled from technology scaling, although at a slower pace due to the many challenges in adapting to degrading device characteristics. Because of the continuing reduction of voltage headroom and intrinsic device gain, high precision analog building blocks have had to adopt higher degrees of design complexity.

\section{THE DIFFERENTIAL AND SINGLE- ENDED VCOs}

A ring oscillator consists of a number of delay stages, with the output of the last stage fed back to the input of the first. To achieve oscillation, the ring must provide two conditions which are summarized as follow. A phase shift of $2 \pi$ and a unity voltage gain at the oscillation frequency. Each delay stage must introduce a phase shift of $\pi=\mathrm{N}$, where $\mathrm{N}$ is the number of delay stages. The remaining phase shift is introduced by a dc inversion [11]. This means that for an oscillator with single-ended delay stages, an odd number of stages are an important condition for the dc inversion. In case of using differential delay stages, the ring can have an even number of stages and that case is happened when the feedback lines are swapped [12]. The topology of the two circuits is shown in Figure 1.

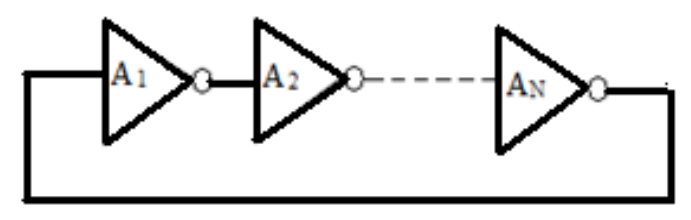

(a)

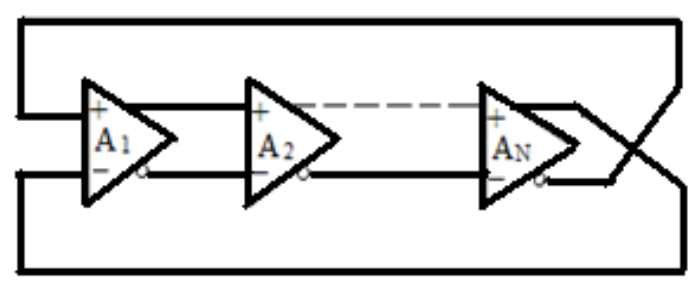

(b)

Fig. 1: Ring oscillator types: (a) single-ended and (b) differential 
one of the simplest way to control the charge and discharge time of an inverter is to control the flow of the current through the inverter, via a voltage controlled current source, as shown in Figure 2. This current source is driven by the control voltage, Vctrl, and the current will determine the charge up and discharge time of the inverter. This topology is called current-starved inverter, as the regular inverter is short of the current they are normally allowed to consume.

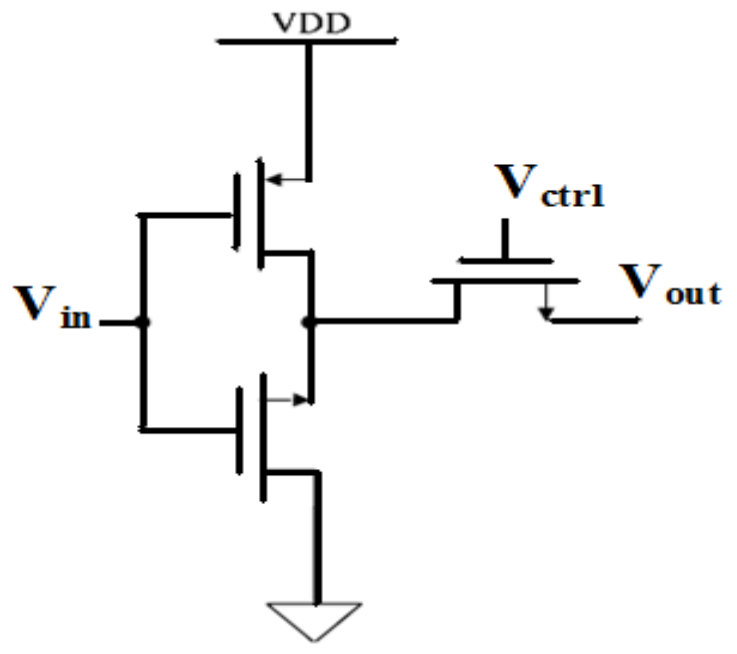

Fig. 2: The current-starved inverter

Differential VCO cells have better oscillation than the singled ended versions and noise performance, due to their current mode logic and native differential noise rejection. In theory, the minimum number of stages required for oscillation of differential cell design is two, as the outputs of the second cell can be crossed to get additional $90^{\circ}$ phase shift. This circuit is shown in figure 3

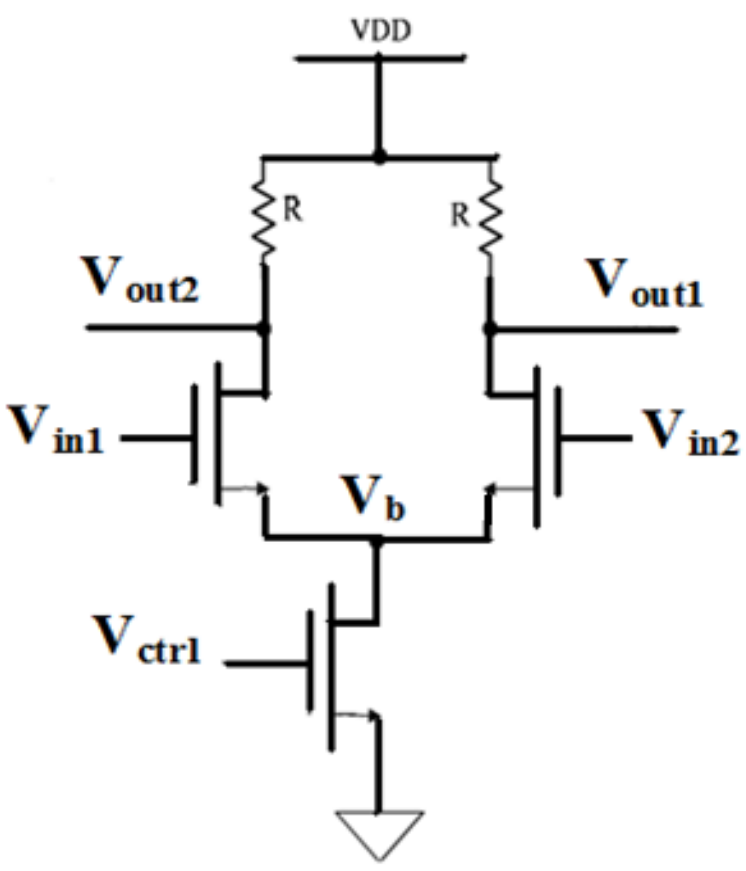

Fig. 3: The Differential VCO with resistive load

\section{THE RING VCO}

It should begin by giving a brief for the advantages of both differential and single-ended topologies. First, differential topology has fast oscillation frequency due to its current mode logic, on the other hand, the output of the differential topology drives only one gate, whiles the output in the singled-ended case, drives both the gates of an NFET and a PFET. This indicates that the output drives as less capacitance as possible to achieve maximum speed.

Second, the current source in the differential design has no major effect on the tuning range, as long as there is a voltage controlled load device, which controls the gain, while the current source in the singled-ended design is important because it is considered the only tuning mechanism [13]. In case of removing the current source and having a voltage controlled load device, this helps in boosting the output swing while maintain the tuning range. This idea is shown through a VCO cell which is shown in figure 4

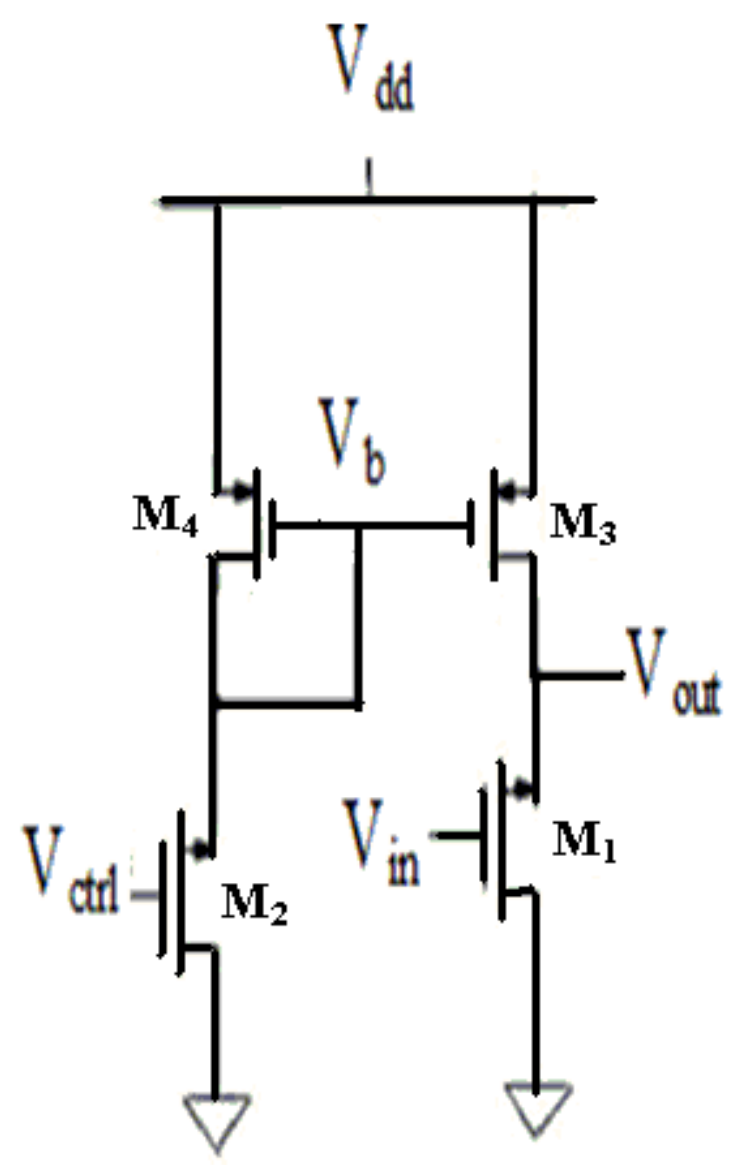

Fig. 4: The Ring VCO Cell Topology

This topology looks very simple. The core "inverter" consists only of a common source amplifier, while the load device is biased by the inverting scheme from the control voltage. As it has seen in the differential pair with PFET loads, this biasing scheme introduces a good idea in producing a large tuning range. In additional to that, the output of the VCO cell drives only another gate, this allows to achieve maximum frequency [11]. However, there are still two old problems within this 
design which are as follow the DC offset problem and small swing at low control voltage problem.

\section{THE MODIFIED RING VCO}

The VCO output swing is minimized as control voltage goes close to zero. This is due to the fact that as Vctrl goes near zero, the voltage at node $\mathrm{V}_{\mathrm{b}}$ becomes close to $\mathrm{V}_{\mathrm{DD}}$. This will put the PFET load into subthreshold region. Though this circuit will still oscillate, but the output swing will be extremely small and cannot drive any outputs. As a result, the VCO does not work at low control voltages. To let the VCO to oscillate at low control voltage, This can be achieved by adding an extra diode connected NFET to the bias circuit, as shown in Figure 5. This diode connected NFET serves as a feedback device that lets the current passes through it whenever the voltage at node $V_{b}$ becomes too high. NFET device can be sized as much smaller compared to the main NFET so that it has little effect when the control voltage is high.

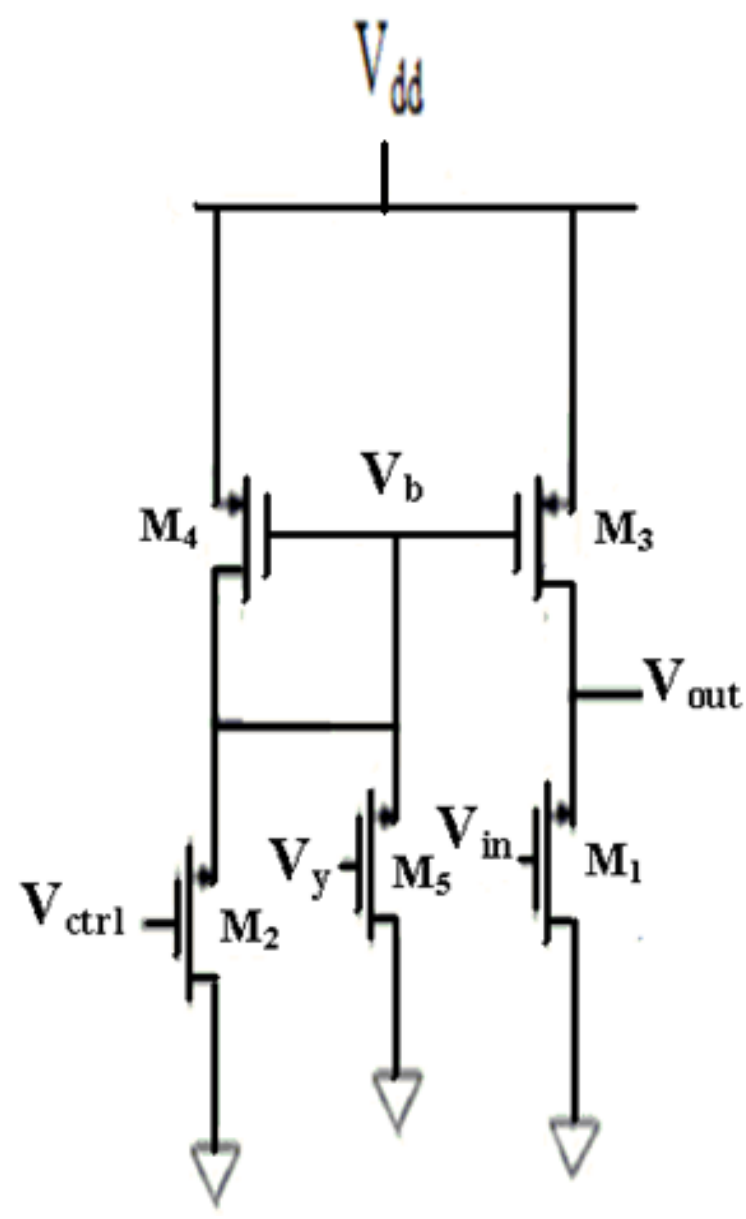

Fig.5: The Modified Ring VCO

\section{The PROPOSED RING VCO}

The proposed circuit shown in figure 6 is based on the benefits of the previous of the two circuits discussed in sections 3 and 4 . In the proposed circuit, M3 and M4 is not connected directly to vdd as the pervious circuit. But it is connected to PMOS transistors (M6 and M7) instead. This connection helps us to obtain large tuning range as the two PMOS transistors decrease the voltage of Vdd before it connects to transistors M3 and M4. This connection also helps in obtaining acceptable phase noise.

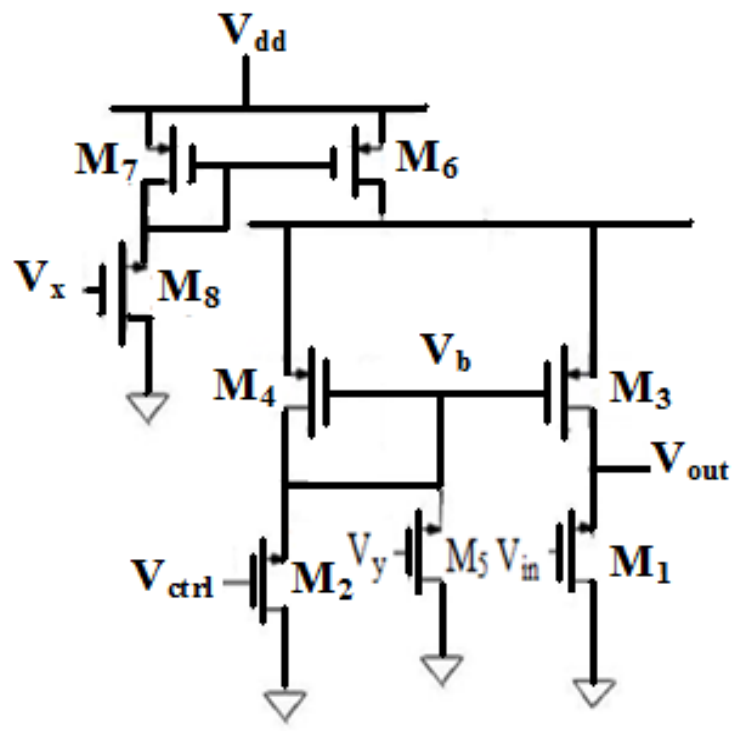

Fig.6: The proposed circuit

\section{ANALYSIS OF THE PROPOSED CIRCUIT}

The proposed circuit is analyzed through the complete circuit shown in figure 7

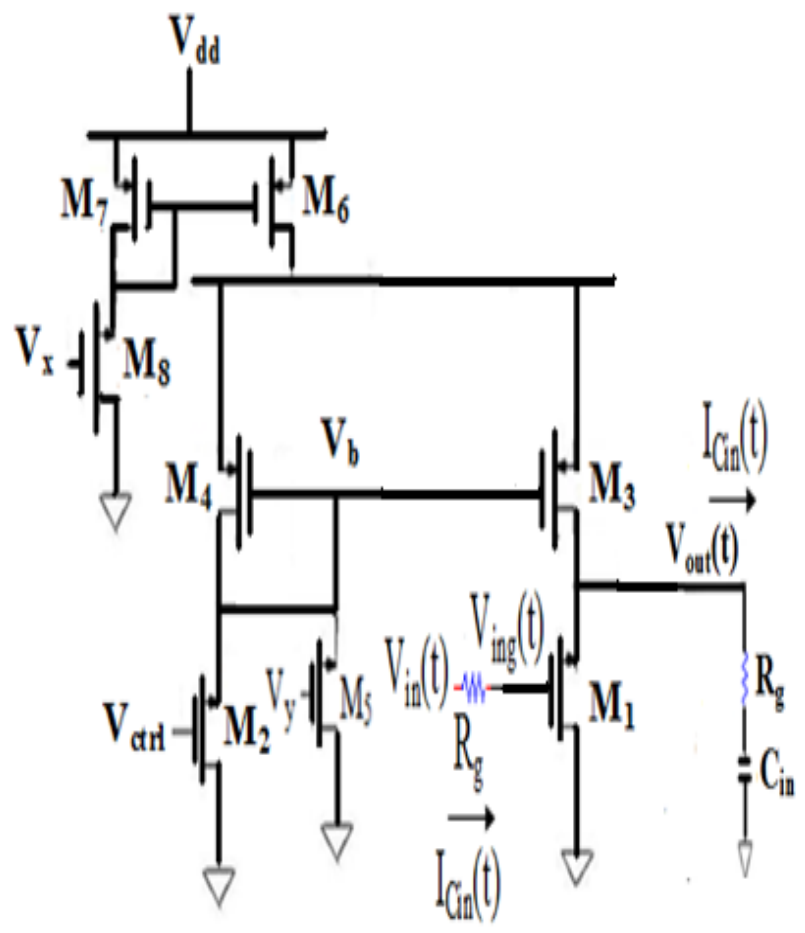

Fig. 7: The proposed circuit with the gate resistance 
With no gate resistance, the voltage on the capacitor Cin is equal to Vout (t). However, with the gate resistance, the voltage on Cin no longer equals Vout $(\mathrm{t})$, and time that the voltage crosses the mid-swing point will shift slightly. This is important because it affects the limits of integration when integrating the current. Therefore, the time that the voltage at Cin crosses the mid-swing point, $\mathrm{V}_{\mathrm{sw}} / 2$, must be determined. This is done by solving (1) for $t_{0}$.

$$
v_{\text {out }}\left(t_{0}\right)-I_{\text {Cin }}\left(t_{0}\right) R_{g}=v_{D D}-\frac{v_{s w}}{2}
$$

Where $I_{\text {cing(t) }}$ is found through KCL. To simplify the expressions, all the parasitic capacitances will be lumped into a term called $\mathrm{C}_{\mathrm{par}}$. Making the substitutions into (1) gives (2).

$$
\begin{gathered}
\sin \left(\pi \frac{2 f t_{0} N-N-1}{N}\right)+2 \pi f R_{g} C_{\text {par }} \cos \left(\pi \frac{2 f t_{0} N-N-1}{N}\right) \\
=0
\end{gathered}
$$

Solving (2) for $\mathrm{t}_{0}$ gives (3).

$$
t_{0}=\frac{\pi N+\pi-a \tan \left(2 \pi f \cdot R_{g} C_{p a r}\right) N}{2 \pi f N}
$$

The argument of $\operatorname{atan}(2 \pi \mathrm{fRgC}$ par $)$ is much less than 1. If $\theta$ is small, then $\tan (\theta) \approx \theta$. Therefore (3) can be simplified to (4)

$$
t_{0}=\frac{\pi N+\pi-2 \pi f \cdot R_{g} C_{p a r} N}{2 \pi f N}
$$

Eq. (4) can be further reduced to (5), which gives the time that the voltage on $C_{\text {in }}$ crosses the mid swing point. This is the time to be used as the lower limit on the integration of the current.

$$
t_{o}=\frac{N+1}{2 N f}-R_{g} C_{p a r}
$$

The subsequent times to be used in the integration are still separated by a quarter period. Using the new integration limits. After substitution and integration, (6) is obtained.

$$
\begin{aligned}
& \frac{1}{2} C_{\text {in }}=\frac{\pi+2+4 \pi f_{1} R_{P} C_{\text {par }}}{8 \pi f_{1} R_{p}} \\
& \frac{+\left(4-8 \pi f_{1} R_{p} C_{\text {par }}\right) \sin (\theta) \cos (\theta)}{8 \pi f_{1} R_{p}} \\
& \frac{-\left(4+8 \pi f_{1} R_{p} C_{\text {par }}\right) \cos ^{2}(\theta)}{8 \pi f_{1} R_{p}}
\end{aligned}
$$

where $\theta=\pi f R g C_{p a r}$ and $R p$ is the equivalent resistance of the PMOS load. Since $\theta<<1, \sin (\theta)$ is $\ll<1$, and these terms become negligible. Also, since $\theta<<1, \cos (\theta) \approx 1$. Making these substitutions results in (7)

$$
\begin{aligned}
& \frac{1}{2} C_{\text {in }}=\frac{\pi+2+4 \pi f_{1} R_{p} C_{\text {par }}}{8 \pi f_{1} R_{p}} \\
& \frac{-\left(4+8 \pi f_{1} R_{p} C_{\text {par }}\right.}{8 \pi f_{1} R_{p}}
\end{aligned}
$$

Solving (7) for $\mathrm{f}_{1}$ gives

$$
f_{1}=\frac{\pi-2}{4 \pi R_{p}\left(C_{\text {in }}+C_{\text {par }}\right)}
$$

The same steps can be repeated to determine $\mathrm{f}_{2}$. The result is given in (9).

$$
f_{2}=\frac{4 \pi I_{s S} R_{p}+N V_{s w}(2-\pi)}{4 \pi N\left(V_{s w}\left(C_{i n}+C_{p a r}\right)+2 I_{s s} R_{g} C_{p a r}\right)}
$$

Averaging $f_{1}$ and $f_{2}$ gives the overall frequency equation

$$
\begin{gathered}
f=\frac{I_{s s}}{2 N V_{s w}\left(C_{\text {in }}+C_{\text {par }}\right)} \times \\
{\left[\frac{1+\frac{I_{s s} N R_{g}}{V_{s w}} \cdot \frac{C_{\text {par }}}{C_{\text {in }}+C_{\text {par }}}\left(\frac{1}{2}-\frac{1}{\pi}\right)}{1+\frac{2 I_{s S} R_{g}}{V_{s w}} \cdot \frac{C_{p a r}}{C_{\text {in }}+C_{\text {par }}}}\right]}
\end{gathered}
$$

Eq. $(10)$ is of the form $(1+a) /(1+b)$ where $a, b<<1$. Therefore $(1+a) /(1+b) \approx 1-(b-a)$. Using this simplification (10) reduces to $(11)$

$$
\begin{gathered}
f=\frac{I_{s s}}{2 N V_{s w}\left(C_{\text {in }}+C_{\text {par }}\right)} \times \\
\left(1-\frac{I_{s S} R_{g} C_{\text {Ppar }}}{V_{s w}\left(C_{\text {in }}+C_{\text {par }}\right)}\left(2-N\left(\frac{1}{2}-\frac{1}{\pi}\right)\right)\right)
\end{gathered}
$$

\section{SIMULATION RESULTS}

The proposed ring vco is simulated via Pspice tool, figure 8 illustrates the input signal in the time domain.

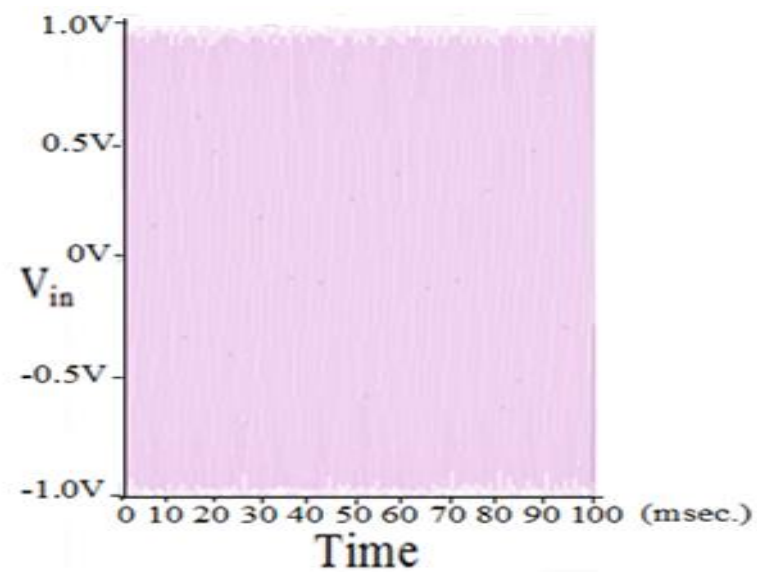

Fig. 8: The input signal 
Figure 9 shows the response of the circuit versus the variation of Vetrl

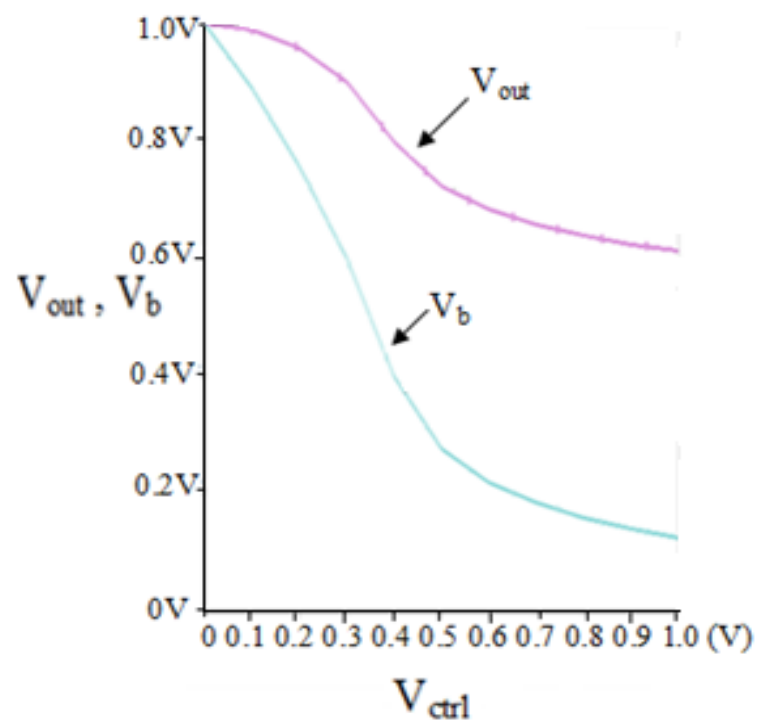

Fig. 9: The response of the circuit versus Vetrl

The conclusion from figure 9 is that $\mathrm{V}_{\text {out }}$ varies from .6v to $1 \mathrm{v}$. This increases the tuning range as $V_{\text {out }}$ Varies linearly with it. This is the good benefit obtained from the proposed circuit. Figure 10 study the oscillation of the proposed circuit in the time domain

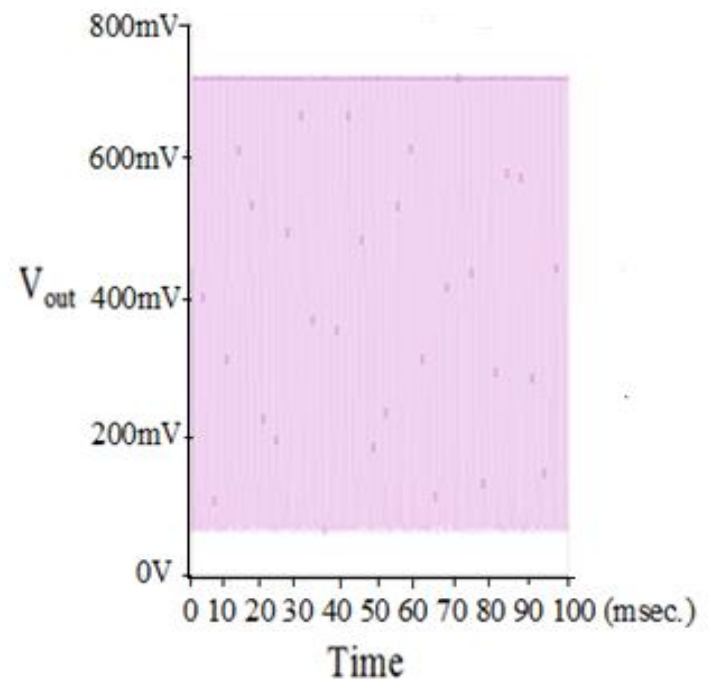

Fig. 10: The response of the proposed circuit in time domain

\section{COMPARISON OF THE RESULTS}

Table 1 demonstrates the comparison of the different researches in ring vco approaches to illustrate the benefits of the proposed work.
Table 1. Comparison between different approaches

\begin{tabular}{|l|l|l|l|l|}
\hline & Technology & Frequency & $\begin{array}{l}\text { Power } \\
\text { supply }\end{array}$ & $\begin{array}{l}\text { Phase } \\
\text { noise }\end{array}$ \\
\hline This work & $\begin{array}{l}65 \mathrm{~nm} \text { SOI } \\
\text { CMOS }\end{array}$ & $\begin{array}{l}2.5 \sim 36.6 \\
\mathrm{GHz}\end{array}$ & $1 \mathrm{v}$ & $\begin{array}{l}-95.5 \\
\mathrm{dBc} / \mathrm{Hz}\end{array}$ \\
\hline $\begin{array}{l}\text { Ring } \\
\text { VCO }\end{array}$ & $65 \mathrm{~nm}$ SOI & $3.2 \sim 26.3$ & $1.5 \mathrm{v}$ & $\begin{array}{l}-97 \\
\mathrm{dBc} / \mathrm{Hz}\end{array}$ \\
\hline $\begin{array}{l}\text { Modified } \\
\text { Ring } \\
\text { VCO }\end{array}$ & $\begin{array}{l}65 \mathrm{~nm} \text { SOI } \\
\mathrm{CMOS}\end{array}$ & $\begin{array}{l}2.8 \sim 28.5 \\
\mathrm{GHz}\end{array}$ & $1.5 \mathrm{v}$ & $\begin{array}{l}-96.5 \\
\mathrm{dBc} / \mathrm{Hz}\end{array}$ \\
\hline
\end{tabular}

\section{CONCLUSIONS}

VCO is the one of the most important building block in modern communication systems. This paper analyzed the design of high performance ring VCO. Through simulation, great results from the proposed design were obtained. The proposed design of ring $\mathrm{VCO}$ was concentrated from maximum oscillation frequency and tuning range perspective. Also the Proposed design achieved a large tuning range with acceptable phase noise and low operating voltage which are good results in the domain of search. This work had positive effect on the study and application of the voltage controlled oscillation in the wireless communication systems.

\section{REFERENCES}

[1] C. H. PARK, O. KIM, B. KIM 2001, "A 1.8-GHz selfcalibrated phase locked loop with preciseI/Q matching", IEEE J. Solid-State Circuits, vol. 36, pp.777-783.

[2] L. SUN AND T. A. KWASNIEWSKI 2011, "A 1.25-GHz 0.35- $\mathrm{m}$ monolithic CMOS PLL based on a multiphase ring oscillator", IEEE J. Solid-State Circuits, vol. 36, pp.910-916.

[3] J. SAVOJ AND B. RAZAVI 2001, "A 10-Gb/s CMOS clock and data recovery circuit with a half-rate linear phase detector", IEEE J. Solid-State Circuits, vol. 36, , pp.761-767.

[4] C. K. K. YANG, R. FARJAD-RAD, M. A. HOROWITZ, 2008, "A 0.5- m CMOS 4.0-Gbit/s seriallink transceiver with data recovery using oversampling" , IEEE J. SolidState Circuits, vol. 33, , pp.713-722.

[5]M. ALIOTO, G. PALUMBO 2010, "Oscillation frequency in CML and ESCL ring oscillators", IEEE Trans. Circuits Syst. I, vol. 48, pp.210-214.

[6] B. RAZAVI 2007, "A 2-GHz 1.6-mW phase-locked loop", IEEE J. Solid-State Circuits, vol.32, pp.730-735.

[7] G. JOVANOVI C, M. STOJ \CEV, Jun 2007, "A Method for Improvement Stability of a CMOS Voltage Controlled Ring Oscillators", ICEST, vol. 2, pp. 715718 .

[8] Huang Shizhen , Lin Wei , Wang Yutong ,Zheng Li, March 18 - 20 2009, "Design Of A Voltage-controlled Ring Oscillator", pp.614-620.

[9] B. Razavi 2003, "Design of Integrated Circuits for Optical Communications",McGraw-Hill. 
[10] N. M. Nguyen and R. G. Meyer May 2002, "Start-up and Frequency Stability in High- Frequency Oscillators", IEEE Journal of Solid State Circuits, vol. 27, pp. 810820 .

[11] S. Docking, M. Sachdev 2003, "A Method to Derive an Equation for the Oscillation Frequency of a Ring Oscillator", IEEE Trans. on Circuits and Systems - I: Fundamental Theory and Applications, vol. 50, 2, pp.259-264.
[12] G. Jovanovic, M. Stojcev, Z. Stamenkovic, 2010 "A CMOS Voltage Controlled Ring Oscillator with Improved Frequency Stability",vol.2,1, pp.1-9.

[13] K. O. Kenneth, N. Park, and D. J. Yang Jun. 2002, "1/f noise of NMOS and PMOS transistors and their implications to design of voltage controlled oscillators", IEEE Radio Frequency Integrated Circuit Symp., pp. 5962. 